\title{
Bonney's Blue Solution in the Treatment of Leprosy.
}

\author{
James LegGate.
}

OTIMULATED by C. S. Ryles' article in the LEPRosy REVIEW of July, 1933, on his results with Brilliant Green and Crystal Violet, I had some of Bonney's Blue solution prepared according to the formula given :-

Brilliant Green, 0.5 grammes.

Crystal Violet, 0.5 grammes.

Absolute Alcohol, 25 c.c's.

Aq. Dist., to 2.500 c.c's.

and selected ten very bad C3 cases for experimental treatment.

Like Ryles I started on open leprotic ulceration (strongly positive) and used the ordinary "Flit" spray pump (as for tannic acid treatment of burns) as the means of application. The solution was sprayed on frequently during the day, and during the night the part was covered with lint soaked in the dye, and covered with water-proof silk. The results were dramatic. The purulent condition subsided in a matter of days, and the sores started healing.

It seemed possible that this was due to a general bactericidal action which was clearing up a secondary infection, so intramuscular injections were given to ordinary hard nodular C3 cases with marked, though not so rapid, effect. In addition, I gave intradermal injections into the nodules. Results in these cases were good. At Mtoko we noted rapid absorption of even firm, hard, fibrous nodules, and do not echo Dr. Ryles' disappointment at this method.

But the quickest results were in the soft purulent node. These were counter-punctured and irrigated through an intradermal needle with a stream of Bonney's Blue Solution. The pus rapidly subsided and the holes scabbed and healed. There was no suspicion of any systemic reaction and, in February the drug was given intravenously in view of the considerably less discomfort afforded the patient. The dose was first 3 c.c's to 5 c.c's, increasing rapidly till 10 c.c's and 12 c.c's were being given weekly.

This has been the procedure up to to-day with the selected cases who are given 12 c.c's weekly and 2 c.c's or 
more into local lesions which are still outstanding. However, the remaining patients (some $\mathbf{5 0 0}$ odd) are so impressed with the results that they are clamouring for the new medicine, and this week it was determined to alternate Bonney's Blue with the iodised esters as routine treatment. This will necessitate reverting to the intramuscular route as being the only safe method for administration by orderlies, on whom the greater part of the work must, of necessity, devolve. One case developed a febrile reaction after an intravenous injection. This turned out to be malarious and yielded to quinine. The dose was halved at the next injection, but after that he was put on a full 12 c.c's dose with no untoward result.

Treatment of the $\mathrm{N}$ type is not so dramatic, though sufficient evidence is not yet forthcoming here. Edges of macules have reacted and subsided, and though no unilateral check has been kept, those particular areas subjected to intensive treatment would appear to show improvement. Whether this is due to the general systemic result of intravenous therapy or a local change it is impossible to say.

It would appear that Bonney's Blue solution is worthy of an extended trial. It may be that its greatest effect will be in countries with ample sunlight, and in Rhodesia, at least, the results have been most encouraging.

Microscopic examination seems to confirm the clinical improvement. Of the ten cases which started Bonney's Blue in January, two in particular, new patients who have had no other drug at any time, show definite fragmentation of bacilli and irregular staining after four months of weekly injections.

Laryngeal, throat and nose conditions have all responded well. One small youth admitted as a last stage C3 on March 20th, with the strained, husky whisper of throat involvement, demonstrated a marked improvement in speech in less than a month. I have not observed this very rapid improvement on admission with any other of the known preparations for the treatment of leprosy, and hitherto I have met no case of those changed over that has not done definitely better on the dye than on iodised esters.

Now that further experiments are to be made over a wider field, it is probable that some small percentage may do better on oil or esters as Ryles found, but up to the present I can only record with satisfaction an apparent superiority of Bonney's Blue over all other forms of treatment. 